\title{
An Evolving Awareness of the Impact of AI and Robotics on the Workplace by Future Employees
}

\author{
Catherine Giunta \\ Seton Hill University
}

\begin{abstract}
This paper summarizes a study of university undergraduate, upper-class status, students about their perceptions of the potential impact of artificial intelligence and robots on the workplace and their careers. The findings are relevant because of the rapid evolution of these technologies and their potentially dramatic impact on the current and future workplace. Job design, career development, and talent development are important considerations. This study can inform the development of presentations and more lectures by professors and companies on this topic. It can help to substantiate the need for proactive, organizational endeavors to prepare managers and employees for transitions that occur due to AI and robotics. Very little research has investigated university students' perceptions of these factors and how it may impact their careers.
\end{abstract}

Keywords: workplace, employees, artificial intelligence, robotics, training, marketing

\section{INTRODUCTION}

Artificial intelligence and robotics are having a dramatic impact on the workplace. This research investigates junior and senior status University students and their perceptions and concerns about artificial intelligence and robotics. As they prepare for their future careers, college students need to have considered what artificial intelligence and robotics are and how they will impact their career tasks in the short run and in the long run. Their career planning in the immediate future and in the long-term time span is discussed. This research shows the need for education and training of future career professionals and managers about the ramifications of this automation.

\section{RESEARCH}

My research will begin investigation of this data and its analysis' impact on university students as current or future full-time employees. Junior and senior university students completed a pre- and postsurvey regarding the use of Artificial Intelligence and robots at the workplace. The pre-survey took approximately 20 minutes. The post survey took 20-30 minutes. The purpose of this study was to research university students' perceptions of artificial intelligence and robots at the workplace. This study can inform development of presentations and more lectures by professors and companies on this topic. Very little research has investigated university students' perceptions of these factors and how it may impact their careers. 
The results were quantitatively analyzed for closed questions or multiple-choice questions. Fill-in the blank, open question data were analyzed using open coding. Open coding will be used to identify themes found in the data (Miller \& Salkind, 2002). The information was grouped using the syntactical, and thematic approaches (Cooper \& Schindler, 2003).

This research was in conjunction with an assigned film, The Bicentennial Man (1999), in the Human Resource Management seminar class. Before the film, and subsequent lecture and discussions, students were given the research pre-survey. The film was shown, and students were quizzed on various aspects, including how Human Resource Management functions. Students were asked the significance of various meeting and conversations with Andrew, the main character, an android purchased as a household robot. The movie's evolution of Andrew as a significant contributor to the household, to a sculptor earning income, to an android who invents life-saving human organs was evaluated. Students analyzed the employee analogies to Andrew being unique, irreplaceable. Another topic for consideration was how machine learning is central to the events of the movie, and researching a current example of machine learning at the workplace. The subsequent ethical issues were analyzed. Working relationships were examined to assist students in shifting to consideration of robots as active "members" of a workplace. Furthermore, students individually stated exactly what impact they believed AI, machine learning, and/or robotics will have upon their career goal.

In today's business world, the use of artificial intelligence and robots is increasing at the workplace to make businesses more efficient and cost-effective. Yet, very limited research has been conducted involving university students. The generational perspective on this topic is significant. Millennials and Gen $\mathrm{Z}$ will be directly impacted by AI and robots in the workplace. Furthermore, Larson (2018) states that "improvement of labor conditions requires governmental and companies to manage any transition... Change is coming" (p.33). Overall, research demonstrates the significance of this topic for business. Because the students, in the HR Seminar utilized in this study, were all Business Administration majors and largely Human Resources and/or Marketing specializations, this research can be relevant to Human Resources and to Marketing educators, and to business Human Resources and Marketing professionals. This research demonstrates the significance of this topic for business users. This is very relevant to events in Business.

\section{FINDINGS}

\section{Artificial Intelligence}

Artificial intelligence is defined as "a machine's ability to perform cognitive functions typically associated with humans, such as perceiving, reasoning, learning, interacting, creating, problem solving" ("An Executive's Guide," para 1). It usually leverages machine learning algorithms to detect patterns and learn how to make predictions and recommendations by processing data and experiences (Dillon, 2020). Although there is not "evidence of progress toward a superintelligence that can outperform humans at any tasks it may choose to undertake" .... there have been Hollywood "blockbusters" that show the "inclusion of human-like features in public AI technology demonstrations". Research shows that new jobs will evolve, as "the labor market constantly evolves in response to automation" (Kaplan, 2017, p 37). AI "will redefine jobs, enhance employee productivity and accelerate workforce development. In fact, skills and culture, not technology, are the biggest barriers to business growth in the AI era. This means chief executives are looking to their chief human resource officers to lead culture change, manage talent and drive down costs" (Britt, 2019, para 1). Satya Nadella, CEO of Microsoft invested 1 billion dollars in a startup company's Open AI technology. According to Open AI's founder, Sam Altman, "our mission is to develop artificial, general intelligence, broad AI systems that can do a lot of tasks at superhuman level. When we have computers that can really think and learn that's going to be transformative" (Kahn, 2020, p 62). Nadella, shortly after he was appointed CEO of Microsoft in 2014, began to reposition the company around artificial intelligence. These companies are competing against Alphabet, Google's parent company, and some other companies to develop the technology that will reshape the business world. There are two types of AI: narrow such as Alexa and Siri, and general AI. It is estimated by "McKinsey Global Institute that the application 
of narrow AI will add 13 trillion dollars to the global economy by 2030, an amount that it says would make the technology more impactful than the steam engine was in the 1800s" (Kahn, 2020, p 63). Some of the progress in AI is a branch called natural language processing (NLP). Examples of this include an algorithm used by Facebook to better identify hate speech and bullying in a variety of languages. It also includes open AI training, a system that has led to an algorithm that can write several paragraphs that are mostly coherent from a human authored prompt of just a few sentences. This could have a noticeable impact on improving digital assistance. Companies that are working on this are looking to target business needs for their discoveries. This breakthrough may also have a notable impact on the employees who previously completed these tasks.

General AI has the goal of making a single piece of software capable of learning almost any task at human or superhuman level. The system would master new skills quickly, for example, by possibly watching a single demonstration or just reading, without needing machine learning. Microsoft and Alphabet each invested in two separate R\&D firms that are largely dedicated to developing advanced stage AI. Facebook has invested in a firm; Chinese Baidu has one lab. Smaller such labs exist at Uber and Salesforce. Many computer scientists feel that general AI is decades away (Kahn, 2020).

The participants in this research demonstrated a more positive view towards the impact that artificial intelligence may have on the workplace after their education about the uses, pros $\&$ cons of AI. There was a statistically significant higher $(\mathrm{p} \leq 0.05)$ rating for the positivity of artificial intelligence after their education/training in an HR Seminar. (See FIGURE 1.)

\section{FIGURE 1 \\ TO WHAT DEGREE DO YOU BELIEVE ARTIFICIAL INTELLIGENCE HAS A POSITIVE IMPACT ON THE WORKPLACE?}

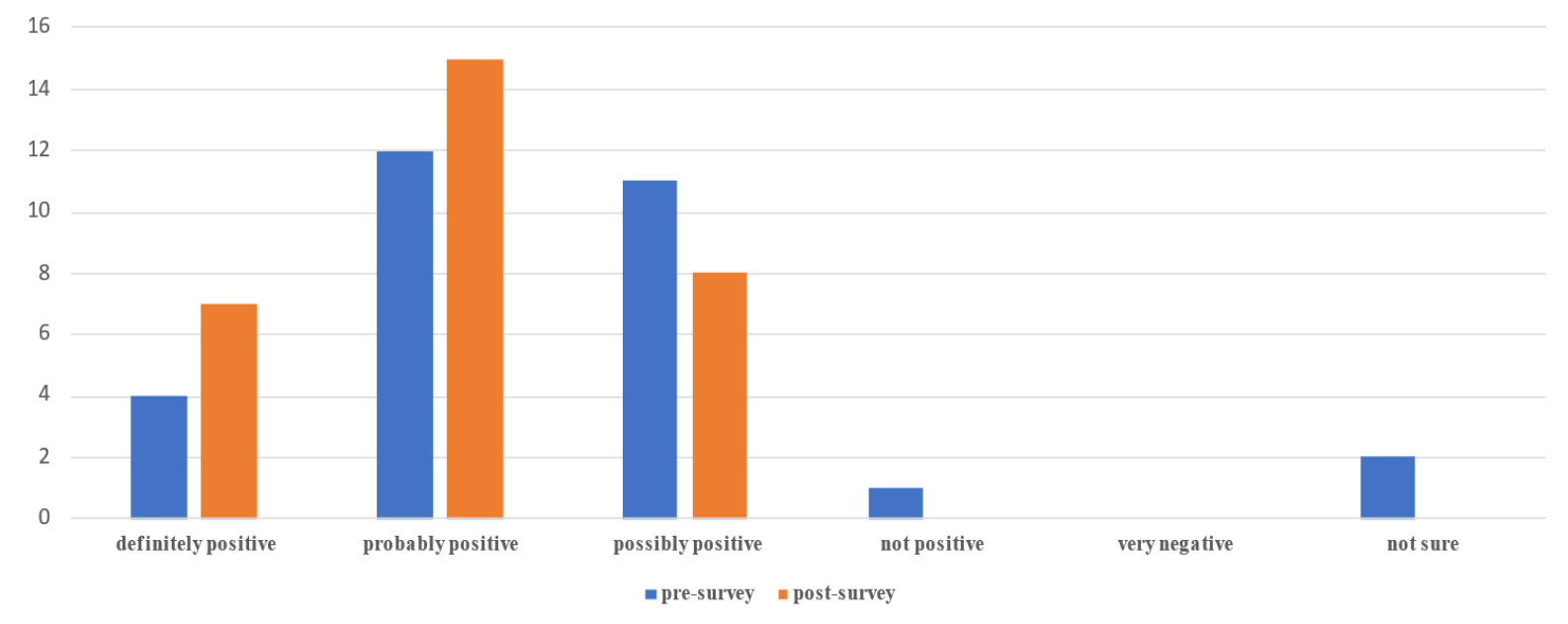

In the post survey most participants believe that artificial intelligence was beneficial to very beneficial. They reported statistically significant $(\mathrm{p} \leq 0.05)$ more positive beliefs about the impact of AI on the workplace. However, a concern still noted by approximately $25 \%$ of the participants was the loss of jobs by humans as a result of artificial intelligence increased use. (See FIGURE 2 below.) This uncertainty about the future of work can potentially influence students' choice of a position with a company after they graduate. Thirgood and Johal (2017) state that labor markets of the future are likely to see a great deal of churn and leave many people facing significant risks on their own (p.25). 


\section{FIGURE 2 \\ TO WHAT DEGREE DO YOU BELIEVE ARTIFICIAL INTELLIGENCE WILL HAVE A DIRECT IMPACT ON YOUR FUTURE CAREER}

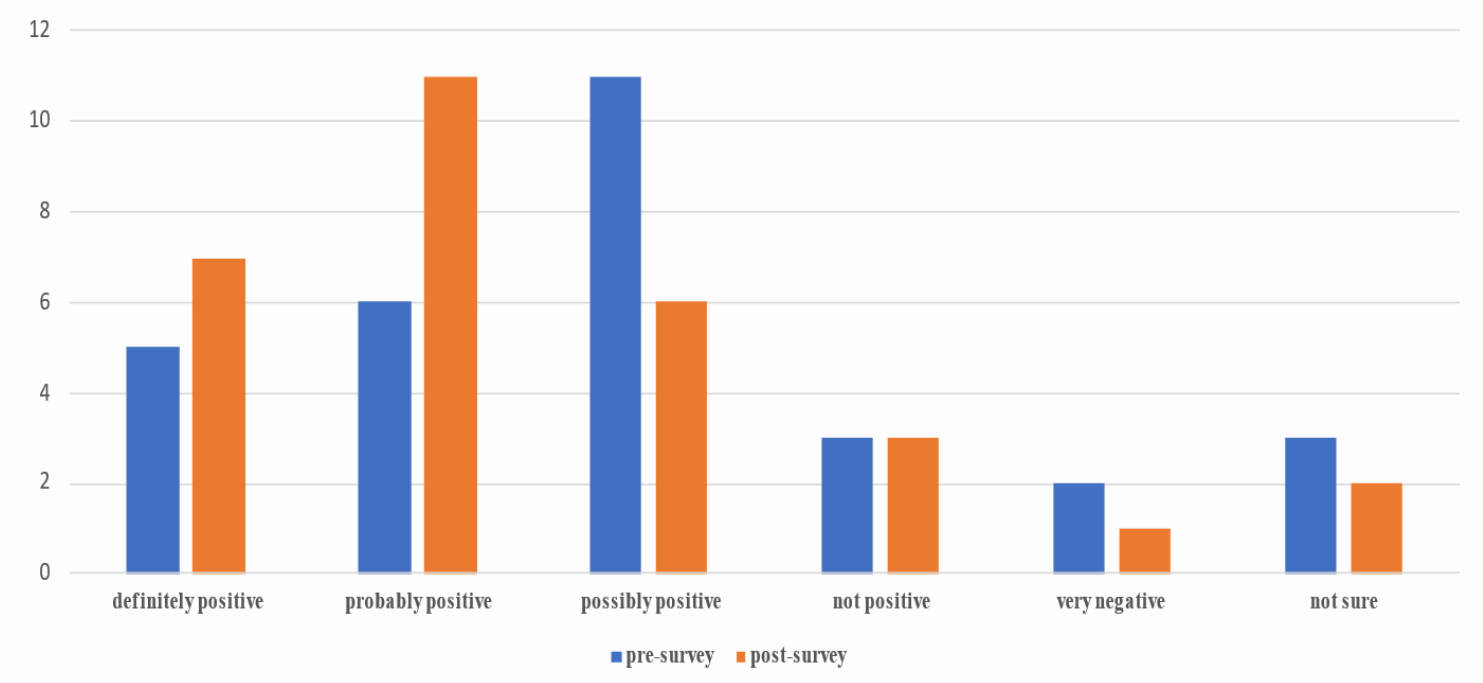

Britt (2019) states that AI can impact productivity positively, for example by reducing day-to-day administrative tasks through apps that make it easier to capture and share expenses and to find colleagues with the requisite skills to perform a project. AI can help in selection by highlighting the optimum candidates for a role, by providing information faster to new employees during onboarding, recommending training and development programs for enhanced staff performance, and by giving managers more data to assist in salary and reward decisions. Additionally, Mauer states that $90 \%$ of the tasks recruiters do will likely be automated in the future (para 2). However, there is "a lot of controversy around Big Data and its potential abuses" Harras, 2021, para 15).

Even though these AI developments may be a concern to job searchers, the loss of administrative work has been occurring for years; "sourcing, screening, assessing and reporting are already highly automated" (Britt, 2019, para 7). Advancements will be made in using predictive analytics routines; yet, hiring high level officials in a company is expected to stay within the responsibility of human beings. Maurer encourages those in the field or entering the field of talent professionals to focus on the human side of the job "building networks, devising strategy, collaborating, and communicating with candidates. Even where it is thought that AI may phase out human bias, we cannot forget that machine learning relies on humans inputting data and crafting algorithms. Therefore, the algorithms can sometime actually reinforce human biases (Maurer, 2017, para 13,14). Furthermore, although marketing hiring was up $6.2 \%$ in a Chief Marketing Officer 2019 report, there was "a 27\% reported increase in implementation of AI or machine learning into companies' marketing tool kits since February of 2018 ... The three uses for AI and marketing are content personalization at $56 \%$, predictive analytics for customer insights at $56 \%$, and targeting decisions at almost 50\%" (Steimer, 2019, p7).

Low barriers to entry in constant, new developments can lead to an increased overall volatility of markets while the higher productivity enabled by technological advancements means firms need fewer employees to get the job done. Many have branded this recent wave of technological change as the fourth industrial revolution. AI is replacing human muscle power in the workplace and brain power (Steimer, 2019 , p. 27). Participants in the study analyzed in this paper noted that artificial intelligence may increase product poverty because of the impact on lower skilled and lower paid jobs. Thirgood and Johal (2017) take this a level higher and note that the jobs at highest risk tend to be those comprised of mostly routine tasks that can easily be performed by a computer, or a machinery automation, and AI. These technologies have 
reached a level at which cognitive human functions can be easily replicated and replaced even in traditionally white collar cognitively-based careers (p.28).

However, research shows that several companies are not properly training their employees for the future including AI's impact. A study entitled, "The state of frontline employee workplace training" found that only $41 \%$ of employees say their employers are training them in preparation for the future. In a variety of industries, employees stated that they only received training a few times a year, sometimes on the wrong things (Newman, 2020, p.10). This paper's research participants were concerned about the impact on human jobs. Although there was a sense that AI can provide safety and help with dangerous jobs, it was also considered not acceptable if human jobs were lost. Mackenzie research indicates that up to $50 \%$ of job tasks can be automated. Additionally, a study by Forbes showed that $80 \%$ of CEOs see artificial intelligence as an important component of their digital transformation strategy (Newman, 2020).

Research by EY shows that artificial intelligence is increasing workplace operational efficiency and decision making (Dillon, 2020). "In theory, automation and artificial intelligence should free humans from dangerous or boring tasks so they can take on more intellectually stimulating assignments, making companies more productive and raising worker wages" (Semuel, 2020, para. 8). The use and expansion of Artificial Intelligence will be determined by humans - managers and customers. Colvin (2018) notes, “A.I.'s eventual uses will be determined largely by market forces... A.I. will be used by companies and consumers for countless practical purposes; most of the modest, and the cumulative effect can't be foreseen" (p.97). There is no doubt that university students will be impacted by artificial intelligence at the workplace. They may eventually need to be re-skilled once they're in the workplace. Research shows that human skills will grow in demand and that fact may calm the students and any individual who is concerned about the impact on artificial intelligence on their careers. McKinsey predicts that "demand for social and emotional skills will grow by $26 \%$ by 2030 across all industries in the United States, while demand for high cognitive skills -- such as creativity, critical thinking, decision-making, and complex information processing -- will grow by $19 \%$ in the same time" (Newman, Ketter, 2019, p.31). By addressing Artificial Intelligence issues in higher-education courses, students will be more aware, and potentially more ready, as the inevitable AI impacts occur. As Gurnani (2019) states, "A monumental change is underway, and artificial intelligence is leading this charge. But we need to remember that humans are ultimately in control" (para. 9).

\section{Robots}

Forms of artificial intelligence, also referred to as robots or bots for short, don't necessarily take up physical space. They are programs, stored on a desktop or a cloud, that have the ability to learn and adapt to different situations as opposed to earlier programs that were more rigid. Because of their learning capabilities, these robots can perform tasks that were deemed impossible by earlier programmers: Robots can write stories. They can understand human speech. They can diagnose a patient better than their own doctor can. An example of software robots making their way into the business world and snagging up repetitive and predictable jobs is the use of robotic process automation (RPA) (McAllister, 2017), which is discussed later.

"Robots have been part of the assembly line doing all kinds of manufacturing for decades" (Colvin, 2018, p. 102). "The unique characteristics of digital enterprises, advancements in automation and artificial intelligence (AI) and on-demand expectations from consumers have further transformed the way we think about work" (Thirgood, Johal, 2017, p. 25). Additionally, “... robots have already taken over many jobs in factories and warehouses .... Even professional services [lawyers] no longer seem safe from the encroachment of increasingly sophisticated artificial intelligence (AI). Yes, "researchers found that...overall employment actually goes up somewhat" (Krakovsky, 2018, p 22). Furthermore, the need for human skills soars, although " 6 of 10 current occupations have more than $30 \%$ of activities that are technically automatable" (Rotman, 2018, p.13).

Researchers predicted the rise of robots will bring about benefits in terms of productivity and economic growth (Taylor, 2019), and most students in this research study commented that the impact will be positive. However, many more participants were unsure about robots' workplace and career impact compared with 
AI (See FIGURE 3). Respondents perceived that robots would increase efficiency and productivity at the workplace. After their education on the subject, they reported statistically significant $(p \leq 0.05)$ more positive beliefs about the impact of robots on the workplace.

\section{FIGURE 3 \\ TO WHAT DEGREE DO YOU BELIEVE ROBOTICS (ROBOTS) HAS A POSITIVE IMPACT OF THE WORKPLACE?}

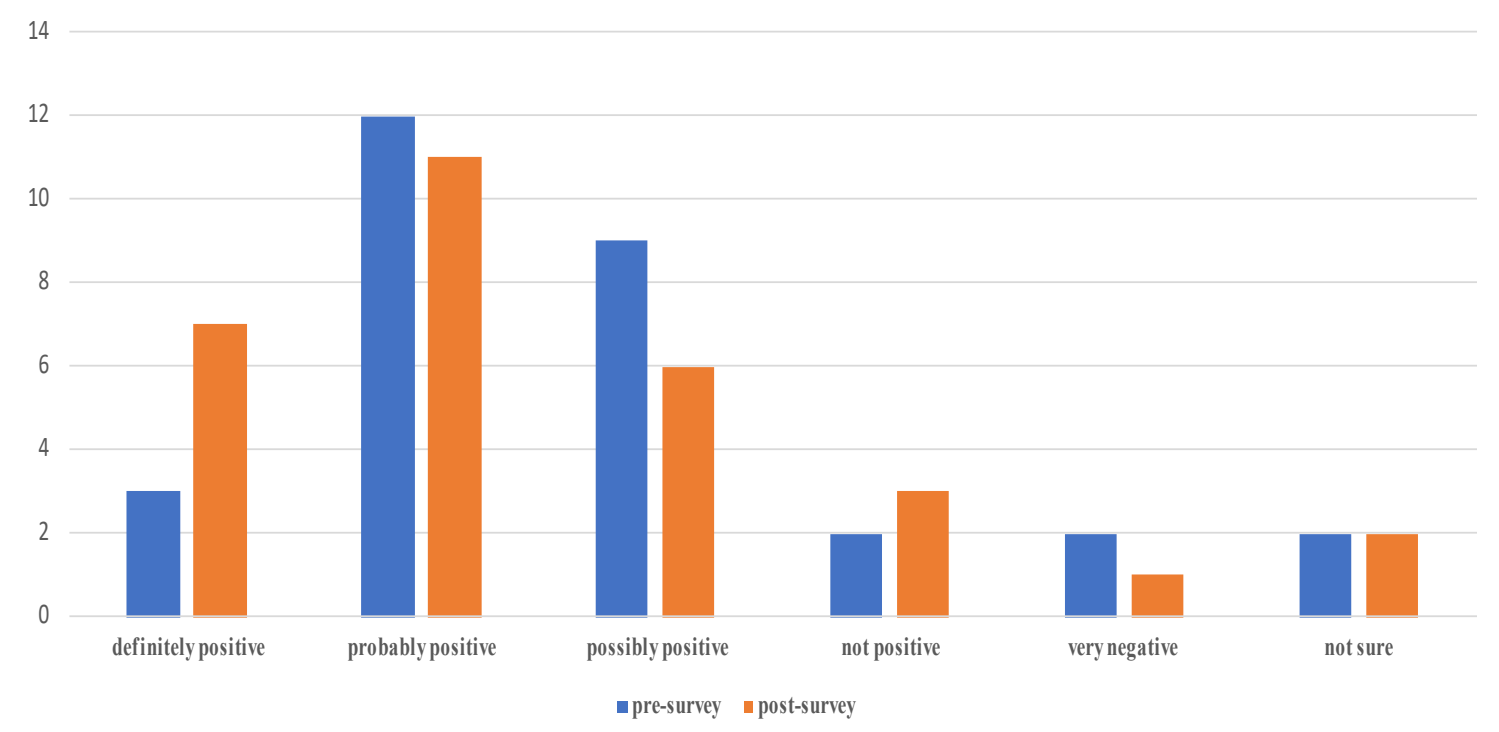

Robotic process automation is a process by which "mundane and boring" jobs, such as data entry and creating spreadsheets, are executed by a robot rather than a human. It frees up the people on the job to do more creative work. If the robot gets too proficient at the job, it could possibly replace its human counterpart altogether (McAllister, 2017). Robotic process automation (RPA) can become very relevant to the considerations of workplace ramifications for managers and employees. It is software that acts as a virtual co-worker in the support of processes. This software assists with basic tasks and therefore reduces the time and cost spent on processes. In manufacturing robots, or collaborative robots work alongside human workers to help the humans complete their tasks. For example, at a Mercedes-Benz plant, robots can more quickly find the relevant part from a huge variety of possibilities. Research is being done on how to teach robots how and when to communicate by reading signals from the humans around them" (Colvin, 2018, p. 102). This may sound like science fiction, but university students will be directly impacted by similar innovations in their workplaces, and possibly their careers. As one research participant stated, "Robots are much more useful than humans would like to admit".

A big advantage of RPA is that it uses existing systems and processes rather than replacing existing HR or payroll systems. Robots can be very helpful because of the data processing that occurs in Human Resource management and marketing (Robotic Process Automation for HR \& Payroll). By being educated about robots at work, positive views were, as one research participant noted, "enhanced". They reported statistically significant $(p \leq 0.05)$ more positive beliefs about the direct impact of robots on their future careers after the Seminar's AI and robotics segment (See FIGURE 4).

Conversely, machines in the past have impacted employment by automating basic tasks, "and while jobs were lost, new jobs were created, providing opportunities for humans to do more valuable things" (Gurnani, 2019, para. 3). Many students in this study were realistic about this point. They recognized that, as one participant stated, they are "emerging everywhere". As one student said, it will continue to develop and increase over time. Robots can make jobs "safer and easier" for humans. For example, there are robots that conduct some of the repairs on the International Space Station for tasks that are deemed to be too 
dangerous for humans. Students also stated that robots can make the workplace safer. A respondent realistically noted that the impact may vary based upon where someone works. Interestingly, among the Human Resource Management majors, there was confidence that in that particular profession the human interaction will still be a strong point of humans. The use of robots in health care was recognized. Additionally, there was recognition that by completing simpler tasks, employees "can focus on more pressing matters".

Despite that appreciation for the value of robots, as with AI, there was a concern on the part of many respondents that robots may take jobs. "Now, manufacturers are clamoring for even more automated machines so they can cope with spiking demand for their products amid a global recovery and a skilled labor shortage". Robots have been called the "perfect pandemic worker" (Popken, 2021, para. 6). Furthermore, "one study estimates that about 400,000 jobs were lost to automation in U.S. factories from 1990 to 2007 . But the drive to replace humans with machinery is accelerating .... The U.S. shed around 40 million jobs at the peak of the pandemic, and while some have come back, some will never return. One group of economists estimates that $42 \%$ of the jobs lost are gone forever" (Semuels, 2020, para 3).

\section{FIGURE 4 \\ TO WHAT DEGREE DO YOU BELIEVE ROBOTS WILL HAVE A DIRECT IMPACT ON YOUR FUTURE CAREER}

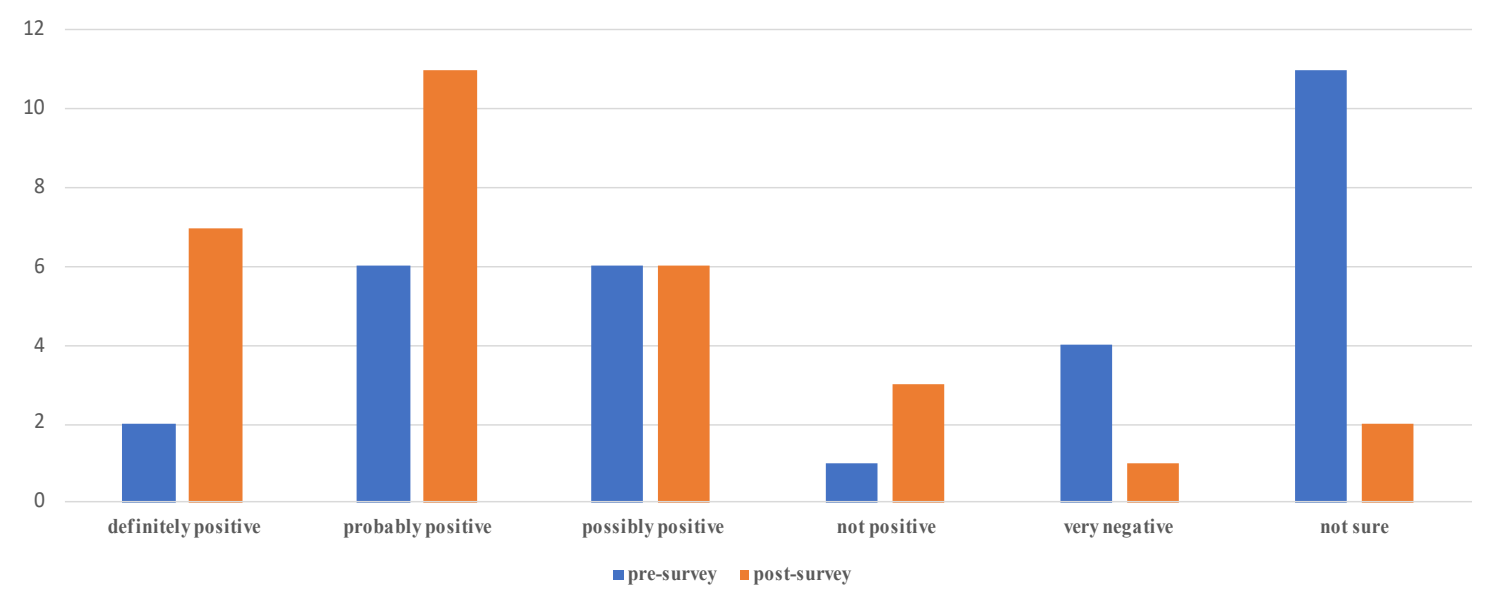

\section{CONCLUSION}

This research investigated university students and their perceptions and concerns about artificial intelligence and robotics. Their views were more positive about the impact of this automation on the workplace and their careers after they were educated about the benefits, and concerns about this evolving situation. This is an important consideration because AI and robots will have significant impact on the workplace and their careers. As the research participants prepare for their future careers, they need to have considered what artificial intelligence and robotics are and how they will impact their career tasks in the short run and in the long run. Their career planning in the immediate future and in the long-term time affected by the technological innovations. This research shows the need for education and training of future career professionals and managers about the ramifications of this automation.

This research shows how valuable it is that students are taught this content as they prepare to enter the workplace as professionals and manager. They can look for the opportunities afforded by AI and robots and implement them. Because of the duality of the benefits and concerns about AI and robots at the workplace, it is essential that employees are open to retraining. A McKinsey study found that "for the period ending in $2030,50 \%$ of current work activities are automatable by technologies that have already been tested and 
found effective" (Kak, 2018, para. 7). The pandemic has accelerated the use of robots and AI, making it more challenging for outplaced employees to be retrained before robots took their jobs (Semuel, 2020). It was predicted that by 2030 , more than 1.5 million jobs will be lost to robots in the United States. Yet, most of these jobs will be low-skills positions. (Taylor, 2019, para.9). In July 2020, two-thirds of companies in a study stated that they were stepping up investment in automation and AI either somewhat or significantly... The scale of workforce transitions set off by COVID-19's influence on labor trends increases the urgency for businesses and policymakers to take steps to support additional training and education programs for workers (Lund, Madgavkar, Manyika, Smit, Ellingrud, Meaney, Robinson, 2021). "Robots could replace as many as 2 million more workers in manufacturing by 2025, Acemoglu found, contributing to wage inequality, a slowdown in labor demand, and an even higher share of GDP going to the owners of capital than labor" (Popken, 2021, para 13). This information highlights the need for the research conducted in this study and the relevance of its findings.

This research is very pertinent to the technological revolution occurring at the workplace and accelerated by companies' responses to the pandemic. Future business professionals and managers can become more knowledgeable about the issues and considerations. They can become more positive about AI's and robot's contributions at work, while also realistically being cautious about the impact on their careers.

\section{REFERENCES}

An Executive's Guide to AI. (n.d.). McKinsey.com.

Britt, A. (2019, October 3). HR Leaders must implement a strategy incorporating future facing technology. The Peoplespace.com.

Colvin, G. (2018, November 1). 25 Ways A.I. is Changing Business. Fortune.com.

Dillon, J.D. (2020, January). The Path to AI. Td magazine. aTD, pp. 25-29.

Gurnani, C.P. (2019, September 30). The AI Revolution is Here. It's Up to Businesses to Prepare Workers for It. CNN.com: Perspectives.

Harras, S. (2021, August 5). As Banks Push AI, Worry about Worsening Inequality Follows. PGe.

Kahn, J. (2020, February). The Quest for Human-Level A.I. Fortune, pp. 62-70.

Kak, S. (2018, February 7). Will robots Take Your Job? Humans Ignore the Coming AI revolution at Their Peril. NBC News.

Kaplan, J. (2017, January). Artificial Intelligence: Think Again. Communications of the ACM, 60(1), 3638.

Krakovsky, M (2018, January). The New Jobs: As Automation Takes on More and More Tasks, What Will Human Workers Do? Communications of the ACM, 61(1), 21-23.

Larson, C. (2018, July). Closing the Factory Doors. Foreign Policy, pp. 32-33.

Lund, S., Madgavkar, A., Manyika, J., Smit, S., Ellingrud, K., Meaney, M., \& Robinson, O. (2021, February 18). The Future of Work After Covid-19. McKinsey Global Institute.

Newman, S. (2020, January). Reaching for Training. TD, p.10.

Newman, S., \& Ketter, P. (2019, November). Future Ready or Not? TD, pp. 28-33.

Popken, B. (2021, April 8). Manufacturers Embrace Robots, the Perfect Pandemic Worker. NBC News.com.

Robotic Process Automation for HR \& Payroll. (n.d.).

Rotman, D. (2018, July/August). Making AI into Jobs. MIT Technology Review, pp. 11-17.

Semuels, A. (2020, August 6). Millions of Americans Have Lost Jobs in the Pandemic-And Robots and AI Are Replacing Them Faster Than Ever. Time.com.

Steimer, S. (2019, October). August 2019 CMO Survey: Hiring, AI on the Rise. Marketing News, pp. 67.

Taylor, C. (2019, June 26). Robots Could Take Over 200 Million Jobs by 2030, Study Claims. CNBC.com.

Thirgood, J., \& Johal, S. (2017). Digital Disruption. Economic Development Journal, 16(2), 23-32. 